\title{
EDUCACIÓN Y ENSEÑANZA EN LAS TESIS DE GRADUACIÓN DE LA FORMACIÓN DE LOS PROFESORES DE EDUCACIÓN FÍSICA EN EL URUGUAY $(1950$ - 1954)
}

\author{
Paola Dogliotti \\ UdelaR - Uruguay \\ paoladogliottimoro@gmail.com
}

\begin{abstract}
RESUMEN
El trabajo aborda las principales discursividades en torno a la educación y a la enseñanza en las tesis de graduación de los estudiantes de la carrera de profesor de educación física impartida en el Instituto Superior de Educación Física de Uruguay en el primer lustro de la década del 50. Se señala la potencialidad de estas fuentes para el estudio historiográfico en el campo educativo. Como parte de la circulación de ideas, teorías y prácticas pedagógicas que muestran las tesis, se aborda la influencia y traducción local de la compleja trama de corrientes psicológicas y filosóficas que conformaron la "Escuela Nueva" y de la perspectiva tecnicista del curriculum de raigambre anglosajona al desarrollo de los fundamentos pedagógicos y didácticos del campo de la educación física.
\end{abstract}

Palabras clave: Escuela Nueva. Curriculum Tecnicista. Educación Física.

\section{EDUCATION AND TEACHING IN THE GRADUATION THESES IN THE CAREER OF PHYSICAL EDUCATION PROFESSORS IN URUGUAY (1950 - 1954)}

\begin{abstract}
The work tackles the main discursivities around education and teaching in the graduation theses of the students in the career of physical education professor given in the Upper Institute of Physical Education of Uruguay in the five first ears of 1950. It signals the potentiality of these sources for the historical study in the educational field. Like part of the circulation of ideas, theories and pedagogical practices that show the theses, its tackles the influence and local translation of the complex plot of psychological and philosophical currents that conformed the "New School", and of the technicist curriculum perspective of Anglo-Saxon origin to the development of the pedagogical and didactic foundations of the physical education field.
\end{abstract}

Keywords: New School. Technicist Curriculum. Physical Education.

\section{EDUCAÇÃO E ENSINO NAS TESES DE GRADUACIÓN DA FORMAÇÃO DOS PROFESSORES DE EDUCAÇÃO FÍSICA NO URUGUAI (1950 - 1954)}

\section{RESUMO}

O trabalho aborda as principais discursividades em torno da educação e ao ensino nas teses de graduación dos estudantes da carreira de professor de educação física dada no Instituto Superior de Educação Física de Uruguai no primeiro lustro da década do 50. Assinala-se a potencialidade destas fontes para o estudo historiográfico no campo educativo. Como parte da circulação de ideias, teorias e práticas pedagógicas que mostram as teses, se aborda a influência e tradução local da complexa trama de correntes psicológicas e filosóficas que conformaram a "Escola 
Nova" e da perspectiva tecnicista do curriculum de raigambre anglo-saxã ao desenvolvimento dos fundamentos pedagógicos e didáticos do campo da educação física.

Palavras-chave: Escola Nova. Curriculum Tecnicista. Educação Física.

\title{
ÉDUCATION ET ENSEIGNEMENT DANS LES THESES DE GRADUATION DE LA FORMATION DES PROFESSEURS D'EDUCATION PHYSIQUE DANS L' URUGUAY (1950 - 1954)
}

\begin{abstract}
RÉSUMÉ
Le travail atteindre les principaux discursives autour de l'éducation et de l'enseignement dans les thèses de graduation des étudiants dans la carrière de professeur d'éducation physique donnée à l'Institut supérieur d'éducation physique de l'Uruguay dans les cinq premières années de 1950. Il signale la potentialité de ces sources pour l'étude historique dans le domaine de l'éducation. Comme une partie de la circulation des idées, des théories et des pratiques pédagogiques qui montrent les thèses, elle aborde l'influence et la traduction locale de la trame complexe des courants psychologiques et philosophiques qui conformaient la «Nouvelle École», et $\mathrm{du}$ curriculum tecniciste des Anglo-Saxons. Origine saxonne au développement des fondements pédagogiques et didactiques du domaine de l'éducation physique.
\end{abstract}

Mots-clés: École Nouvelle. Curriculum Tecniciste. Éducation Physique.

\section{INTRODUCCIÓN}

Este trabajo forma parte de una investigación mayor que trata sobre las discursividades ${ }^{1}$ de la formación de los profesores de educación física en el Instituto Superior de Educación Física $^{2}$ del Uruguay $(1948$ - 1970). Se analizan especialmente aquí los discursos en torno a la educación y a la enseñanza presentes en las tesis de graduación de los estudiantes en el primer lustro de la década del 50. A partir de este análisis se presenta como problema central la mirada sobre la profesión que fue tejida por los estudiantes: ¿cómo entendían su formación, cuáles eran las principales características que debía tener un buen profesor de educación física? ¿a qué

\footnotetext{
${ }^{1}$ A partir del aporte teórico del análisis del discurso francés se entiende a las discursividades como "el punto de encuentro de una actualidad y una memoria" (Pêcheux, 1990, p. 17); el entrecruzamiento de proposiciones de apariencia lógicamente estable, susceptibles de respuestas unívocas y formulaciones irremediablemente equívocas (Pêcheux, 1990, p. 28). A partir de aquí se busca encontrar los diversos anudamientos y quiebres discursivos, sus contradicciones y rupturas.

${ }^{2}$ El Curso de Profesores de Educación Física fue creado en el año 1939 en la órbita de la Comisión Nacional de Educación Física (CNEF) que fue fundada en el año 1911 en el segundo gobierno del presidente José Batlle y Ordóñez para encargarse del desarrollo de la educación física a nivel del sistema educativo, federativo, plazas de deportes y otras dependencias estatales en todo el territorio nacional; a fines del año 1952 se le otorga a la institución donde se desarrollaba el Curso para la Preparación de Profesores de Educación Física el nombre de "Instituto Superior de Educación Física" (ISEF). Durante todo el siglo XX ISEF dependió de la CNEF que a su vez dependía a nivel ministerial de los diversos modos en los que se llamó el Ministerio de Educación y Cultura. Recién en 2006 pasa a la Universidad de la República.
} 
autores, materias y profesores del curriculum vivido seleccionaron con mayor frecuencia para dar cuenta de estos aspectos?

En la búsqueda realizada, dentro del período de nuestra investigación, se encontró un corpus de veintinueve tesis ubicadas en la Biblioteca de ISEF $^{3}$. Se encontraron tesis producidas hasta el año 1954. Debido a una resolución de la CNEF a fines del año $1954^{4}$, este fue el último año en que se cumplió la reglamentación presente en el decreto del 3 de mayo de 1939 de creación del Curso para la Preparación de Profesores de Educación Física que establecía que para obtener el título de profesor de educación física el estudiante debía elaborar una tesis. El Reglamento de ISEF del año 1952, firmado a máquina por el Jefe de Estudios Porf. Alberto Langlade, dedica los artículos 7 y 8 a las tesis y expresa:

Art. 7.- El último trimestre de tercer año, el alumno deberá dedicarse a la confección de su tesis. Para ello presentará al Jefe de Estudios un bosquejo del tema que piensa desarrollar. Si a criterio de éste el tema presenta valor lo aprobará y designará al alumno un profesor guía que lo aconsejará. Indicará conveniencias en el enfoque, bibliografía, etc.

Si el tema presentado no llenara las exigencias mínimas, se indicará al alumno la conveniencia de buscar otro tema, pudiendo sugerirle los que juzgue interesantes para el Curso.

Art. 8.- La Tesis debe ser presentada en un original y dos copias, escritas a máquina, doble espacio, en papel satinado corto y encuadernadas en papel y tela de color marrón, marrón oscuro. (ISEF, 1952, p. 3).

Tanto la supervisión inicial del tema que debía ser aprobada por el Jefe de Estudios, como el acompañamiento por parte de un docente y el tipo de presentación dan cuenta de la importancia otorgada en su momento a la realización de una tesis. Por otra parte, otro aspecto a destacar es la supervisión institucional en el tema elegido por parte del Jefe de Estudios. Esto da cuenta de la impronta que Alberto Langlade le imprimía a la carrera, quien tenía la última palabra en la aprobación del tema de tesis. Nada se dice sobre el mecanismo de su aprobación luego de su entrega.

A continuación se muestra un cuadro con la cantidad de tesis encontradas por año y se los compara con la cantidad de profesores titulados en esos años.

\footnotetext{
${ }^{3}$ Es importante destacar que no se encontraban catalogadas, estaban dispersas entre diversos documentos que habían sido trasladados de la biblioteca de la CNEF. Su sistematización requirió de un gran trabajo. Se agradece la colaboración brindada por el personal de Biblioteca de ISEF.

${ }^{4}$ En las últimas sesiones del año 1954, la CNEF adoptó la siguiente resolución: "Se suprimió la presentación de la denominada "Tesis" a los Egresados del Instituto Superior de Educación Física, la que será exigida posteriormente como condición indispensable, habilitante para el ascenso a la Categoría superior" (CNEF, 1955, p. 47).
} 
Cuadro N. ${ }^{\circ}$ 1. Cantidad de tesis y de profesores titulados discriminados por año (1951 - 1954).

\begin{tabular}{|c|c|c|c|c|c|}
\hline & $\mathbf{1 9 5 1}$ & $\mathbf{1 9 5 2}$ & $\mathbf{1 9 5 3}$ & $\mathbf{1 9 5 4}$ & Total \\
\hline $\mathbf{N .}^{\circ}$ tesis & 3 & 0 & 6 & 19 & $28^{5}$ \\
\hline N. ${ }^{\circ}$ titulados & 6 & 1 & 8 & 46 & 61 \\
\hline
\end{tabular}

Fuente: Elaboración personal a partir de ISEF (2014) y el corpus de tesis encontrado en la Bibliotecas de ISEF.

Se presenta una cierta relación proporcional entre la cantidad de recibidos y el número de tesis halladas por año. Se constata que se lograron conservar el $46 \%$ de las tesis elaboradas. Aparentemente fue la generación del 51 la última que elaboró tesis para la obtención del título.

Se detallan a continuación las 29 tesis clasificadas cada una por su tema principal ${ }^{6}$ en 8 agrupamientos, se discriminan en algunos casos las temáticas específicas dentro de ellos y se muestra entre paréntesis el número de tesis dentro de cada uno.

1. Deportes (6): voleibol (1). básquetbol (1), fútbol (1), natación (1) y atletismo (2).

2. Gimnasia (4): escuelas y métodos (1), en grandes aparatos (1), femenina moderna (1) y educativa (1).

3. Área Biológica (9): entrenamiento (2), higiene escolar y corrección postural/educación física en el obrero (4), alimentación (1), respiración (1) y biotipología (1).

\section{Recreación y campamentos (4).}

5. Educación, educación física y el rol del profesor de educación física (3).

6. Psicología y educación física (2).

7. Historia de la educación física (1).

8. Arquitectura y educación física (1).

Las tesis en su mayoría están bien escritas, con buena sintaxis y ortografía, la mayoría con un estilo académico de la época ${ }^{7}$, otras tendientes a uno de tipo ensayístico. La mayoría presenta una extensión cercana a las 100 páginas, con índice y bibliografía. Muestran a través de citas y bibliografía un amplio manejo bibliográfico y un conocimiento directo de autores del campo de la educación física y sus diversas prácticas corporales, la educación, la psicología, las ciencias biomédicas, entre las más destacadas, si bien se agregan dentro de la bibliografía los apuntes de clases de varios de los profesores a cargo de los cursos. Se percibe a través de su lectura capacidad para citar, analizar y relacionar autores y para la elaboración personal de sus

${ }^{5}$ El número da 28 y no 29 porque en una de las tesis encontradas el año de entrega estaba realizado por el autor a modo de firma al final de esta y no fue legible el último dígito del año (195?).

${ }^{6}$ Muchas tesis abarcaban más de una temática, se optó, a partir de su lectura, por ubicarla en la temática más trabajada o que era abordada en forma principal.

${ }^{7}$ Las citaciones y bibliografía en su mayoría carecen de año de edición, editorial y número de págína las que son textuales. Se presenta en general el nombre del autor y el titulo de la obra citada. 
puntos de vista. Otro aspecto a destacar es que la mayoría de las tesis citaban textos en varios idiomas extranjeros: en mayor proporción en inglés y portugués; algunos en francés y en menor medida en alemán.

Las tesis en general muestran el optimismo de una joven generación, nueva carrera que recién había comenzado, hacía apenas una década de creada. Varias de ellas presentan una crítica al estado de la educación física en el país en los diversos niveles del sistema educativo, a la situación de la CNEF y en especial al estado crítico a nivel edilicio y de recursos del ISEF. Algunos agradecen al inicio, la buena formación recibida por algunos de sus profesores.

En general las tesis parecen haber sido escritas en el sentir de la generación adulta (si bien en esto también puede influir el hecho que algunos estudiantes cursaban con mayor edad a la mínima requerida al finalizar los cuatro años de estudios secundarios, que rondaban los 16 años). Eso lo podemos relacionar, como dice Markarian $(2004)^{8}$, a que la generación del 50 a diferencia de la del 60 todavía no se había revelado ante las opciones de sus padres.

[...] [de] la lectura de boletines o gacetas estudiantiles de la época, ya sea de asociaciones gremiales o de grupos de alumnos liceales. Llama la atención la escasez de interrogantes y demandas sustancialmente diferentes de las que animaban a autoridades y padres, cuando no se comprueba una total adhesión a las actitudes y propuestas de esos adultos. Se distinguía roles según la edad, pero la fuerza explicativa de corte generacional no solía aparecer en las reflexiones sobre el tema, incluso en los pocos casos en que provenían de los propios jóvenes. [...]

Aparentemente, la <cultura adolescente $>$ que surgió en los tempranos cincuenta en Estados Unidos y Europa no impactó inmediatamente en Uruguay. [...] No fue sino hacia finales de la década que los adolescentes uruguayos comenzaron a adherir tímidamente al rock y a diferenciarse por su vestimenta, modismos, reacciones y lugares de encuentro. [...]

La metáfora de la crisálida resume adecuadamente la representación más extendida sobre los adolescentes uruguayos de los tempranos cincuenta: no eran vistos como personas completas, con una $<$ vida propia $>$ (ni siquiera por ellos mismos), sino como $<$ adultos incompletos>. (MARKARIAN, 2004, p. 243-244).

A partir de todos los aspectos desarrollados anteriormente podemos decir que las tesis de los estudiantes proporcionan para la investigación historiográfica otro tipo de mirada, narrativa y de acercamiento al objeto de estudio y a los hechos. La percepción por parte del estudiante a punto de egresar que sintetiza desde su punto de vista los conocimientos adquiridos en sus tres años de carrera nos parece un material de sumo interés para el objeto de la presente investigación. La voz de los estudiantes se muestra en varios trazos de la escritura de la tesis, muchas veces valorando la formación que recibió, otras señalando sus aspectos más críticos,

\footnotetext{
${ }^{8}$ Investigación desarrollada en Uruguay a partir de entrevistas realizadas entre noviembre de 1996 y enero de 1997 a siete hombres y ocho mujeres nacidos entre 1932 y 1940 en diversos sitios de Uruguay. Se cruzan las entrevistas con el análisis de datos del contexto social en que vivieron.
} 
desde una mirada joven y mostrando el impulso juvenil y el desafió de comenzar a transitar los primeros años de la profesión elegida.

El análisis de las tesis también nos permiten entender las principales concepciones, puntos de vista, modos de pensar, cómo sentían su profesión cuestión central para tejer y entender las discursividades de la formación desde la mirada peculiar de los estudiantes a punto de egresar. La tesis eran entregadas luego de haber culminado y aprobado todas las unidades curriculares del plan de estudios.

Por otra parte, otro aspecto relevante al que pudimos acercarnos a través de este tipo de fuente, es a los autores y tipo de bibliografía utilizada para el trabajo, ya sea a través de citas directas o como material de consulta pudimos acceder a cuáles fueron los autores, libros, revistas, manuales, temas, docentes y perspectivas teóricas más relevantes.

Se analizan para este trabajo, de acuerdo al problema seleccionado, en especial las tesis que abordan la educación, la enseñanza y sus métodos, y sus relaciones con el campo de la educación física.

\section{EDUCACIÓN Y ENSEÑANZA EN LA TEORÍA DE LA ENSEÑANZA-APRENDIZAJE}

El trabajo aborda las principales concepciones de educación y enseñanza presentes en las tesis analizadas y cómo estas configuran una discursividad de la teoría de la enseñanzaaprendizaje desarrollada fundamentalmente a lo largo del siglo XX.

\footnotetext{
La teoría de la enseñanza-aprendizaje, ya de marcado sesgo psicologista, implica un viraje esencial del saber (aun como "conocimiento") al sujeto psicológico, colocando a éste en la posición central de proceso objetivable y controlable, transformando a la Didáctica en una rama de la Psicología Aplicada (¿Psicología de la Educación?). Aunque pueda parecer un "embolsado" demasiado ecléctico, todas las construcciones en teoría didáctica centradas en la enseñanza-aprendizaje como parámetro estructurador importado del género próximo de la psicología (psicogenéticas, histórico-culturalistas, conductistas o cognitivistas), se inscriben dentro de esta productividad discursiva. En gran medida, en estos enfoques predomina el aprendizaje, que sustituye al método, y se reduce a la enseñanza a un mero instrumento de intervención. (BEHARES, 2005, p. 12; cursiva del autor).
}

Esta discursividad encierra las afectaciones y traducciones al campo pedagógicodidáctico de los variados tipos de teorías psicológicas, bien diversas en relación a cómo conciben el sujeto, el lenguaje, el tiempo y el saber. Pero todas ellas mantienen ciertas características comunes en relación a estas cuatro categorías: el sujeto se presenta en forma psicológica, uno, de la conciencia o la conducta y en las antípodas de un sujeto barrado, el 
lenguaje es un instrumento de transmisión lineal, trasparente y comunicacional, el tiempo es cronológico y el saber es del orden del conocimiento objetivable y reproducible.

Dentro de la discursividad de la teoría de la enseñanza-aprendizaje se ubican dos configuraciones discursivas: la didáctica psicologizada y curricularizada o tecnicista (BORDOLI, 2005). En la primera, a diferencia del siglo XIX y anteriores, a partir de la didáctica comeniana (BORDOLI, 2005) donde había una centralidad en la trasmisión de conocimientos,

\begin{abstract}
las operaciones de análisis y fragmentación ya no serán los del objeto de conocimiento sino las del sujeto de aprendizaje. El axioma madre sobre el que se construirán los diversos enunciados didácticos es la concepción de desarrollo. [...] $<$ En este momento el centro de la reflexión didáctica, estará en los sujetos de aprendizaje, en la posibilidad de intervenir, regular, graduar sus procesos. Ya no bastará con desagregar a los diversos saberes en átomos de conocimiento, ahora será necesario desagregar al sujeto en sus etapas evolutivas, así como en sus diversas "capacidades" o "conductas" o "competencias $>$. (BORDOLI 2004b9 apud BORDOLI, 2005, p. 21; cursiva de la autora).
\end{abstract}

Dentro de esta discursividad, con diferentes énfasis y enfoques encontramos el desarrollo de la Escuela Nueva o Activa ${ }^{10}$ que tiene como centro el partir del interés y necesidades del educando, la idea de naturaleza, el aprendizaje activo en su centro y el desarrollo integral del individuo, entre otros aspectos que analizaremos, siendo sus inspiradores, Rousseau, Pestalozzi y Dewey entre los más relevantes.

La configuración discursiva de la didáctica curricularizada o tecnicista, tiene su punto de inflexión con la obra de Tyler (1949) Principios básicos del Curriculum, que dio surgimiento a la teoría curricular que invadió y sustituyó, fundamentalmente en los países de habla anglosajona, la educación y la didáctica por el campo del curriculum. A partir de aquí se desarrolló la Tecnología Educativa ${ }^{11}$ y la Planificación Educativa. En nuestro análisis se muestra, fundamentalmente en tesis que trabajan con bibliografía de corte anglosajón, la presencia de esta discursividad. No debemos olvidar que la educación física en nuestro país, a

\footnotetext{
9 "Crisis de la educación y reformas. Los discursos didácticos actuales", en Memorias del Primer Congreso Nacional.: La enseñanza y el aprendizaje en contextos educativos; debates actuales. Org. Signo, IMM, octubre 2004.

${ }^{10}$ Es importante destacar en este punto que según Caruso (2001, p. 93 - 94) el movmiento pedagógico renovador de finales de siglo XIX y de las primeras décadas del XX, denominado "Escuela Nueva", "Escuela Activa" o "Nueva Educación" estuvo conformado por una compleja avalancha de experiencias, propuestas, métodos y articulaciones en un escenario pedagógico internacional con traducciones diversas en Latinoamérica, apoyado científicamente fundamentalmente por la psicología así como por una nueva filosofía de la educación que combinaba movimientos del campo filosófico del fin de siglo europeo con características profundamente espiritualistas y críticas.

${ }^{11}$ Según Diaz Barriga (1991, p. 15) "la adopción de la tecnología educativa, como opción instrumental, científica y como expresión de una pedagogía industrial, permite < garantizar> una formación de acuerdo a una nueva racionalidad: la formación eficiente de recursos humanos para el desarrollo industrial".
} 
partir de la creación de la CNEF, en 1911, tuvo una gran influencia de Estados Unidos, fundamentalmente a través de la llegada de misioneros de la YMCA (DOGLIOTTI, 2015) ${ }^{12}$.

Este nuevo enfoque rompe con las lógicas desarrolladas en torno al saber y/o al sujeto que habían estructurado los discursos didácticos. El nuevo enfoque para pensar lo didáctico tiene cuatro características primordiales: exterioridad, centralidad en lo instrumental, minimización de los saberes y como efecto una noción de sujeto "pragmático". [...] El meollo de lo didáctico se representará en los instrumentos y recursos que se utilicen. A su vez, los saberes, los conocimientos ocuparán un lugar secundario, en tanto que lo medular se ubicará en las actividades de los sujetos de aprendizaje. (BORDOLI, 2005, p. 21-22, la cursiva es de la autora).

\section{EL ESCOLANOVISMO: UNA DIDÁCTICA/PEDAGOGÍA PSICOLOGIZADA}

En la mayoría de las tesis la educación era entendida como el mejor modo de intervención en el proceso evolutivo del educando, debía tener a este en su centro. "El deber primordial del Profesor ante su clase es hacerse amar, orientando la educación a impartir hacia los principios modernos de la ciencia ${ }^{13}$; el estudio del niño, diversidad de los procedimientos empleados para hacer surgir de cada ser un elemento personal" (DE MARÍA, 1954, p. 79). Apoyado en la ciencia del conocimiento del niño, la psicología, el profesor seleccionaba los procedimientos que más se ajustaban a la edad y sexo del niño. "La Educación Física es parte integral de la educación; y el profesor encargado de impartirla, tiene tanto o más responsabilidad del proceso evolutivo de la personalidad del alumno, como el llamado de "aula" o enseñanza común” (BUSCH, 1954, p. 3).

Esto no implicaba que se desconociera el valor del conocimiento a impartir por parte del docente, "el ideal del Maestro, el buen Profesor, es aquel que conoce a fondo la materia que enseña, a fondo, también, la naturaleza del niño, que ama la enseñanza y la niñez, y se hace amar por ella" (DE MARÍA, 1954, p. 112). Conocimiento y sujeto, ambos aspectos eran importantes, pero el peso de la psicología que científicamente descubría la naturaleza del niño, era central y conducía el proceso pedagógico de enseñanza-aprendizaje. "“"El objetivo de la educación es dirigir el desarrollo armónico, natural e integral del educando durante toda su faz evolutiva" (DE MARÍA, 1954, p. 12). Los significantes armónico, natural, integral, desarrollo,

\footnotetext{
${ }^{12}$ Para un análisis de los modos y tipos de influencia de los Estados Unidos a través de la Young Men Christian Asociation (YMCA) en la educación física en Uruguay se recomienda Craviotto; Malán (2014); Dogliotti; Malán (2015); Malán (2017); Scarlato (2018). Para estudios de su influencia en Argentina, Brasil y México se recomienda Ainsentein; Elías (2018); Da Silva; Linhales (2018); Da Silva (2017), Linhales (2015), Martínez Moctezuma (2015).

13 "La valorización individual. La cual debe ser fundamento de toda pedagogía racional de la ciencia de la educación" (ACHENBACH, 1954, s/p).
} 
evolución, se articulaban en torno a la idea de naturaleza y de ciencia, así se citaban a pensadores como Spencer, Dewey, Rousseau, Pestalozzi.

Apelando al supremo juicio imparcial de la naturaleza; nos acogeríamos a las teorías ya expresadas por Rousseau, Pestalozzi y Spencer, sobre la educación del niño, caracterizadas todas ellas en la $<$ vuelta a la naturaleza $>$.

Pestalozzi, le concede a ésta, la facultad de regir el desarrollo armónico: mental y físico.

Spencer pone de manifiesto, como mejor método de educación aquel que más se le aproxime; considera que la conservación de la salud es uno de nuestros deberes y establece la existencia de una moralidad física.

Cuando Rousseau nos dice: $<$ Si usted desea cultivar la inteligencia de su alumno, cultive lasda fuerzas que deben regirla. Ejercite su cuerpo continuamente, hágale fuerte y sano, para que pueda ser inteligente y razonable, déjelo trabajar, déjelo hacer, déjelo correr, déjelo gritar, déjelo siempre en movimiento, déjele ser hombre de vigor que pronto se hará de razón> se podría incluir la frase de tiempo en tiempo. (ACHENBACH, 1954, s/p).

Es a partir de Rousseau que luego Dewey desarrolló en su filosofía de la educación, el concepto de experiencia que parte de esas premisas. "Educación es la reconstrucción o reorganización de la experiencia, que se añade a la significación de dicha experiencia y que aumenta la habilidad para dirigir el curso de la experiencia ulterior" (BENTANCOR, 1954, p. $1)$.

La educación física se configuraba como el mejor modo de llevar adelante estas máximas. Pero naturaleza no significaba la ausencia de límites, de disciplina, sino que estaba unido a la racionalidad científica y la educación física acompañaba ese proceso.

\footnotetext{
Si bien es cierto que la ciencia más sabia es la naturaleza, es evidente, sin embargo, que recién en este momento, el fin utilitario, científico y verdadero, que persigue el ejercicio físico, toma real incremento en la clase de gimnasia. [...] Es ahora cuando la psicología se debe adaptar al movimiento y no al juego libre e imaginativo del niño. [...] La educación: al adaptar al individuo a la vida en sociedad. Esta adaptación pone freno a las manifestaciones naturales del espíritu para reorganizarse dando pauta a una conducta racional. (ACHENBACH, 1954, s/p).
}

Por momentos el sentido de naturaleza se asocia a la ciencia y por otros se opone a esta. Esto traduce las dos lecturas que se han hecho de Rousseau, una más cercana a una naturaleza romántica y asocial, y otra asociada al desarrollo científico y social.

El escolanovismo se basó en los autores mencionados anteriormente para desarrollar y fundamentar sus experiencias educativas. Entre sus ideas centrales estaba el reconocer la espontaneidad del niño y oponerse a la "escuela tradicional o autoritaria"14.

\footnotetext{
14 “Antiguamente educar era someter a los educandos a una instrucción uniforme, reduciendo todas las vocaciones a una común” (DE MARÍA, 1954, p. 14).
} 
Los sistemas autoritarios "modernizando la definición: dictatoriales" llevan en esencia el aniquilamiento de la energía y espontaneidad del niño, no permitiéndole captar el valor intrínseco del bien, apreciándolo unicamente por el "premio o castigo" sin reconocer las ventajas de este proceder. (ACHENBACH, 1954, s/p).

La escuela activa se oponía a un modelo de instrucción disciplinaria inculcada a través del temor, y el profesor de educación física poseía amplias condiciones para oponerse a ese modelo a través del respeto de la esponeidad y la naturaleza del niño. "El Profesor alegre y comunicativo será comprendido mejor por su educandos que aquél que emplea métodos y disciplinas severas" (DE MARÍA, 1954, p. 65).

Como hemos podido comprobar a través de algunos casos vividos de los innumerables
que se reproducen diariamente, esa disciplina que implanta el Profesor de Educación
Física, no es aplicada con la energía y la frialdad de quien tiene que dominar un grupo
imponiendo respeto a toda costa, haciéndolo reaccionar bajo la forma hueca de las
órdenes impartidas con severidad, inculcando el temor entre sus dirigidos. [...] Es por
el contrario la disciplina que ordena sus impulsos, que regula sus actos moderándolos
por la vía de la ejecutoria correcta, pero sin llegar nunca a cortar la espontaneidad, ni
sus reacciones naturales y propias de la edad. Para conseguir ésto es necesario
conquistar al niño, y para conquistarlo es menester gozar de su presencia, sentirla
intensamente. (MAUAD, 1953, p. 19).

La centralidad en el niño y el establecimiento de un vínculo cercano entre docente y educando fue clave en esta pedagogía que criticaba el intelectualismo y la instrucción sin educación.

Actualmente se instruye más que se educa. Tanto la educación moral como la física fueron sacrificadas en pro de la educación intelectual. Es así que la máxima de fuego con que Rabelais fustigó a sus contemporáneos "ciencia sin conciencia es la ruina del alma” encuentra hoy también su justa aplicación. (ACHENBACH, 1954, s/p).

En este contexto discursivo se realiza una cita a Mantovani, pedagogo escolanovista argentino, quien crítica la educación instrumental y utilitaria y propone "acentuar el carácter ampliamente humano de la educación", una "escuela primaria formativa, no meramente informativa” (MAUAD, 1953, p. 20). Se menciona explícitamente a la pedagogía activa o la nueva educación:

Es necesario hacer de cada profesor un colaborador social, se le debe dar [...] sobre todos las normas de una pedagogía activa, vinculada a los intereses y realidades del niño (DE MARÍA, 1954, p. 95).

La nueva educación debe proponder al desarrollo integral de la personalidad, debe favorecer la solidaridad y la cooperación social, debe desarrollar en un amplio ambiente la libertad, no debe nunca apartarse del contacto íntimo con la naturaleza y debe ser una función independiente de intereses momentáneos. (DE MARÍA, 1954, p. $15)$. 
Se destacan dos componentes centrales de esta "nueva" o "activa" pedagogía, además de las ideas de naturaleza, integralidad, solidaridad y cooperación social ${ }^{15}$, libertad y espontaneidad desarrolladas anteriormente:

1. el partir de los intereses y de la realidad del educando

2. la idea de niño activo en oposición a pasivo

Con relación al primero, se presenta el siguiente concepto de interés:

\begin{abstract}
El interés es el estado del alma que se produce cuando hay íntima concordancia entre la naturaleza del individuo y el objeto que se impone a su atención. Hay verdadero interés cuando el "yo" se identifica con una idea o un objeto; y esto va a suceder cuando ha encontrado en ese objeto o en esa idea, el medio de expresarse. (DE MARÍA, 1954, p. 84).
\end{abstract}

El interés se asocia a la idea de naturaleza y pone en su centro, como estado del alma, la identificación entre el sujeto y el objeto o idea, que se manifiesta a través de la expresión. El niño debe ser "movilizado" de algún modo por el objeto de enseñanza. Es interesante ver cómo en una de las tesis que refería a la enseñanza del basquetbol femenino se señalaba como parte de las indicaciones generales para la enseñanza de los juegos y deportes, el "desarrollar el interés en el juego, llamando la atención sobre los puntos en contacto que tiene con otros deportes practicados anteriormente" (PREOBRAYENSKY, 1954, p. 16). El partir de los conocimientos previos del alumno se configuró a partir del desarrollo de las psicologías fundamentalmente cognitivistas, en especial, la de Ausubel, en una de las máximas del aprendizaje significativo. Para que esto se pueda dar era necesario amalgamar las necesidades sociales con los intereses y caracterísiticas psicológicas de los grupos y los individuos.

Es necesario que la misma [Educación Física] esté orientada apreciando el nivel de calidad física de cada sociedad en que va a actuar, estar de acuerdo con las necesidades actuales de la misma sociedad [...] adaptándose a las características psicológicas generales y particulares de los individuos, adecuándose a las características de cada grupo social, recordando que el individuo es formado por la sociedad y al mismo tiempo es formador de ella; donde se parte de un todo sin perder su libertad. (DE MARÍA, 1954, p. 22).

La Educación Física, como toda Educación, debe atender los principales intereses del elemento a educar $[\ldots]$ como las mismas posibilidades e intereses que el medio social en que actúan y se desenvuelven. (DE MARÍA, 1954, p. 26).

El Profesor de hoy tiene que ser un estimulador del interés, un despertador de necesidades intelectuales y morales con entusiasmo como virtud principal. (DE MARÍA, 1954, p. 45).

\footnotetext{
${ }^{15}$ A diferencia de la formación de profesores de educación fisica en el INEF Manuel Belgrano de Buenos Aires (1938 - 1967) donde el componente de las pedagogías comunitaristas fue central (AISENSTEIN; FEGUIN, 2016, p. 16), en el caso que estamos analizando si bien está presente tuvo menor ascendencia. Quizás en el caso Argentino se acentuaba por sus características de internado.
}

Rev. Iberoam. Patrim. Histórico-Educativo, Campinas (SP), v. 4, n. 1, p. 122-143, jan.jun. 2018 
El hincapié en el desarrollo de necesidades es parte de la argumentación, en este punto es necesario destacar que la teoría de las necesidades ${ }^{16}$ tiene una base fisiológica y orgánica sobre lo social, es de algún modo una manera de soslayar que las necesidades son construidas, producto de las relaciones histórico-sociales, incluso aquellas más necesarias como el alimento.

Respecto al segundo aspecto o componente, se destaca que el educador es "la fuente de estímulos más activa sobre el niño durante su vida escolar" (DE MARÍA, 1954, p. 116). Este debe brindarle una serie de "centros de interés" como fuente de estímulos para que el niño en forma activa descubra su propio conocimiento. Su papel es más el de un guía que de un trasmisor. El profesor de educación física tiene a través del juego un instrumento central para el desarrollo de esta pedagogía.

El juego educa e instruye a la vez que formula hábitos y proporciona conocimientos. De acuerdo a "la ley de actividad infantil" que se aplica tanto a la vida fisiológica como a la psíquica, tenemos que, el juego no sólo desenvuelve el físico, sino que, "cuando las aptitudes físicas son activas, también lo son las intelectuales y morales" (Pastrascoiu). (ACHENBACH, 1954, s/p).

Así se establecía la importancia de la educación física en la etapa infantil, en tanto permitía el "desarrollo de las propiedades de la inteligencia por medio de ejercicios físicos" (ACHENBACH, 1954, s/p), en tanto "toda la vida activa muscular, es el dictamen del conjunto de los factores de la inteligencia" (ACHENBACH, 1954, s/p).

En sintonía con los principales axiomas de la Escuela Nueva, en varias tesis se presentaba una dura crítica a la educación física al servicio del deporte espectáculo, al logro de "records" o exclusiva formación de campeones, lo que Vaz Ferreira ${ }^{17}$ (1922, p. 33) ${ }^{18}$ denominó "recordismo especialista ${ }^{19 "}$ ya que esto estaba en contra del desarrollo armónico e integral del cuerpo infantil.

\footnotetext{
${ }^{16}$ Se hacer referencia a la Pirámide de las Necesidades desarrollada por Abraham Maslow, psicólogo estadunidense, en su libro Una teoría de la motivación humana de 1943.

${ }^{17}$ Carlos Vaz Ferreira (1872-1958) es considerado uno de los filósofos uruguayos más reconocidos en la primera mitad del siglo xx por su vasta obra en relación con temáticas filosóficas y su interés en problemáticas educacionales. Desde fines de siglo fue catedrático de filosofía en la Universidad. Fue miembro del Consejo Directivo de Instrucción Primaria (1900-1915) y Decano de Preparatorios de 1904 a 1906. Entre los años 1929 y 1943 ocupó con interrupciones el cargo de Rector. Desde 1946 hasta su fallecimiento dirigió la Facultad de Humanidades y Ciencias, cuya creación impulsó desde el rectorado.

${ }^{18}$ Se citó por el año en que Vaz Ferreira dictó la conferencia en el ámbito de la CNEF y no por el año de edición.

${ }^{19}$ Entre la serie de errores y males que conlleva el "recordismo especialista" Vaz Ferreira destaca: su oposición al juego, la falta del desarrollo de fuerza equilibrada, la unilateralidad y grado de fuerza excesiva que ocasionaba desequilibrios y se oponía a la salud, el solo hecho de atender a la juventud abandonando la adultez y la vejez, perjuicios físicos en etapas posteriores y abandono de los "normales" y los "débiles" por solo atender a los excepcionales (DOGLIOTTI, 2015, p. 104 - 106). Para un análisis del discurso vazferreiriano en relación a la educación física consultar Dogliotti (2015, p. 100-108).
} 
El papel del Profesor frente a la sociedad no es el de simple conductor de individualidades "campeonistas" hacia el logro de "records" o performances extraordinarias, sino el proporcionar a los alumnos un desenvolvimiento armónico del cuerpo y del espíritu, formando un ser activo y resuelto que va a colaborar en el servicio de todos. (DE MARÍA, 1954, p. 48).

Otras argumentaciones se centraban en la crítica al deporte espectáculo y al papel de la prensa, en el contexto del Uruguay recién obtenido el título de campeón mundial de fútbol.

\begin{abstract}
Y si el deporte constituye la plataforma del récord y del negocio a veces poco limpio del espectáculo deportivo; si estimula y cultiva el narcisismo, la vanidad personal y desdén por las cosas del espíritu, si llena las páginas de la prensa con lisonjas y vulgaridades, si provoca rozamientos y despierta las pasiones patrioteras y belicosas de la multitud en torneos internacionales, (recuérdese escenas al triunfo de nuestros futbolistas), ésto se debe a la poca educación deportiva, a la baja calidad tanto de parte de atletas como por parte del público.

Ahora bien, el deporte es sí, libre de la obsesión del record, en su primitivo y verdadero carácter, llena y embellece enorme parte de la verdadera Educación Física. No constituye propiamente un medio de desarrollo físico conveniente al niño como base de la educación escolar, sino que constituye más bien un medio de perfeccionamiento físico cuyo rol está al final, como consagración de la Educación Física. (BENTANCOR, 1954, p. 37-38).
\end{abstract}

En estos años, todavía se insistía desde argumentos inspirados en la Escuela Nueva y en el pensamiento vazferreiriano, que el deporte no era un medio recomendado de educación física sino luego de culminada la etapa escolar, al iniciarse la adolescencia.

Otros argumentos aludían a la oposición entre "recordismo especialista" y salud:

El deporte deja de ser deporte, porque toda actuación que oblige a sufrir desgaste físico y perjuicio orgánico, no es una exaltación de la educación física, sino el abuso inadecuado de unas facultades, siempre limitadas, que se ponen al servicio de un espectáculo antes que de la salud del cuerpo y de la mente. (PREOBRAYENSKY, 1954, p. 136).

Si bien los temas de educación fueron los que predominaron frente a los de didáctica o enseñanza, especialmente dos tesis trataron los principios generales de esta última. La enseñanza era entendida fundamentalmente como proceso de aprendizaje, confundiéndose la primera en el segundo y este último en términos de evolución orgánica. Así se presentaba en una tesis dedicada al tema de la enseñanza de la natación: "Analizamos primeramente los principios generales de la enseñanza, principios aplicables no solo a la natación, sino a todo proceso de aprendizaje que pretenda una obra de real valor" (BUSCH, 1954: 1). En este contexto la enseñanza y sus métodos era entendida como un sistema que "se debe graduar teniendo en cuenta su edad y sexo" (BUSCH, 1954, p. 2), según las características orgánicas o biológicas del cuerpo y la mente en cada edad y en cada sexo. 
Principios generales de la enseñanza

¿Cuál es el mejor método a seguir? Aquel método racional, basado en una serie de razones pedagógicas y psicológicas, que se apoyan además en las condiciones del medio, clima, edad, sexo y cualidades individuales de los alumnos, El método no será nunca único ni inflexible, pero debe ajustarse siempre a ciertas condiciones imprescibles. (BUSCH, 1954, p. 7, el subrayado es de la autora).

La enseñanza se entendía como el método racional, informado por las ciencias psicológicas y pedagógicas, y las variables fundamentales que lo determinan se centraban en las caraterísticas del alumno (sexo, cualidades individuales y edad) y del medio. A diferencia de la didáctica comeniana donde el método era único, aquí se señalaba que no será único ni inflexible.

Los principios generales de enseñanza de la educación física citados en varias de las tesis $^{20}$, aludían por un lado, a la determinación del cuerpo orgánico, y por el otro, a lo que se entendía por principios metodológicos a cumplir en su enseñanza, nuevamente la enseñanza se mimetizaba, confundía con el método y menos se preocupaba por el saber a trasmitir que por las características orgánicas tanto mentales como corporales de los educandos. Estos eran:

a) continuidad (acompañar al ser humano en todas las etapas evolutivas, la actividad debe evitar toda interrupción que perturbe los procesos funcionales y psicoespirituales estimulados);

b) progresividad (gradación pedagógica en la dificultad e intensidad de la actividad a realizar, a partir de lo psíquico o físicamente simple a lo complejo);

c) variabilidad (forma de crear nuevos centros de interés que contemplen los deseos generales de la clase, los especiales de cada individualidad y los fundamentos técnico-pedagógicos de la disciplina);

d) alternancia (cada clase, así como el plan anual o semanal, obedecerán a una curva regular, ascendente e ininterrumpida, que debe ser respetada por razones fisiológicas; la gradación de la intensidad del esfuerzo, como el descanso alternado de las diferentes masas musculares, nos llevará a evitar un gran déficit de oxígeno al finalizar la clase, ya que la alternancia en la actividad permitirá recuperar la cuota de oxígeno necesaria en el decurso de la lección) (BENTANCUR, 1954, p. 28-29; BUSCH, 1954, p. 7-9).

Se mezclaban aspectos de una didáctica comeniana relativos a la gradación en grado de complejidad, de lo simple a lo complejo, con principios pedagógicos escolanovistas, como el trabajo por centros de interés, la centración en el alumno, el partir de los deseos e intereses del grupo y el individuo, aspectos específicos de una didáctica psicologizada que pone el centro en

\footnotetext{
${ }^{20}$ Estos principios son extraídos del apartado 1 “Educación Física y Gimnástica” del Capítulo I del Manual de Didáctica de la Gimnástica de Langlade (1956) que trata de fines, medios y principios fundamentales de la educación física y citados por Preobrayensky (1954); Busch (1954) y Bentancor (1954).
} 
el aprendizaje y en acompasar el método al desarrollo de las etapas evolutivas del educando, y finalmente, aspectos centrados en el desarrollo fisiológico del cuerpo.

\section{LA ENSEÑANZA COMO DIDÁCTICA CURRICULARIZADA O TECNICISTA}

En sintonía con la inclusión en esta década de aspectos relativos a la enseñanza o didáctica en el Programa de Pedagogía ya que no había una unidad específica para ello, la siguiente tesis ubica estos aspectos en un capítulo titulado "Pedagogía".

\section{Consideraciones generales sobre la enseñanza}

Se ha demostrado claramente que las experiencias son básicas en el aprendizaje. Las personas reaccionan ante un estímulo y esta forma de reacción se aprende. La teoría de las conexiones en la enseñanza, es una de las más aceptadas por los educadores. Esta teoría dice que el aprendizaje se produce por medio de una serie de conexiones nerviosas que se hacen mucho más permeables por la repetición de la respuesta. Una creencia muy común en todos los sistemas de psicología es que la conducta de un individuo está influida o condicionada por sus experiencias pasadas (PREOBRAYENSKY, 1954, p. 8)

Es clarísimo en esta cita como la enseñanza se sustituye por el aprendizaje o es definida desde la teoría de las conexiones desarrollada por Skinner que estudia desde la teoría conductista cómo aprenden los individuos. Varias son las tesis que lo citan. La base de esta teoría es la experiencia entendida como acumulación de estímulos y las respuestas a ellos por parte de los individuos como conducta; la físiología y lo orgánico (como conexiones neuronales) es su sustrato epistemológico.

\footnotetext{
Como dice Skinner, experimentar es percibir, sentir, pensar, tener propósitos, crear y obrar. Por lo tanto, la experiencia que produce directa o indirectamente una conducta y un servicio mejorados, es la más valiosa, tanto para el individuo como para la sociedad. Implica además un proceso de individualización y de socialización que lleva consigo crecimiento y conducta $[\ldots]$

Ahora bien, considerada como producto, la Educación es el resultado alcanzado por el desarrollo y la experiencia o actividad del aprendizaje. (BENTANCUR, 1954, p. 12).
}

En esta teoría del aprendizaje la conducta es efecto de lo exterior al individuo, la experiencia que proporciona estímulos. Lo observable y medible a través de la ciencia experimental constituye la base epistemológica de esta teoría y el énfasis es puesto en el producto o resultado y las actividades de aprendizaje. En esta perspectiva el método lejos de ser entendido en función del saber a enseñar, es instrumental y una guía para provocar el aprendizaje en función de los objetivos planteados. La importancia en la planificación por objetivos conductuales que se desarrolló en el ámbito educativo a partir de esta teoría y el 
surgimiento de la teoría curricular que pone el acento en la planificación en función de una racionalidad técnica es central en esta perspectiva.

\section{Métodos de enseñanza}

Método es la forma en que se hace una cosa. Cuando hablamos de métodos de enseñanza, queremos significar procedimientos que se han usado repetidamente y que representan una técnica familiar que ha ido mejorándose en la práctica y por la experiencia. Los métodos pueden valorarse en términos de principios fundamentales, guías y por su efectividad en acompañar los objetivos de la educación. (PREOBRAYENSKY, 1954, p. 10).

El método pensado desde el aprendizaje era procedimental y en función de las conductas a lograr en los individuos; así se soslayaba el saber a enseñar. Por eso al detallar sus principios fundamentales se destacan cualidades como rapidez, eficiencia y efectividad, partir de los intereses de los educandos, experiencia, resultados, demostración, disciplina, control y manejo de la actividad.

\footnotetext{
Principios fundamentales del método, procedimientos adecuados en todo método de enseñanza:

$1^{\text {a }}$ Deben efectuarse adaptaciones de acuerdo con la diversidad de intereses y capacidades que se encuentran en los diferentes individuos

$2^{\circ}$ El aprendizaje de los alumnos debe proyectarse en términos de su pasado experimental, ya que las nuevas experiencias se interpretan a la luz de las experiencias pasadas

$3^{\circ}$ Hay que mantener a los alumnos interesados, receptivos y voluntarios para que el aprendizaje sea así más rápido, más eficiente y más efectivo

$4^{\circ}$ Los alumnos deben saber claramente cuales son los resultados que se esperan, y han de estar al tanto, a intervalos regulares, de los progresos que han efectuado $5^{\circ}$ Los alumnos deben tener oportunidades para demostrar lo que han aprendido $6^{\circ}$ Debe haber una forma de disciplina, control y manejo, apropiada a las actividades que se están practicando. (PREOBRAYENSKY, 1954, p. 10-11).
}

El aprendizaje motor es el que mejor se adapta a la teoría conductista, en tanto exige la ejecución de movimientos a alta velocidad y en forma automática. Entre sus principios se destaca la importancia en la demostración y el modelo, captación del interés, centralidad en la actividad, práctica y ejecución, reforzamiento de las conductas deseables, eficiencia y efícacia, rapidez y automatismos, resultados, importancia en la organización de la clase.

Desarrollo de las habilidades fundamentales o motoras:

Como resultado de estudios que se efectuaron sobre la psicología del aprendizaje motor, se han aceptado ciertos principios que pueden aplicarse al desarrollo de las habilidades. Algunos de los más importantes:

1. El alumno debe estar interesado y en estado de buena voluntad [...]

2. Debe efectuarse una demostración clara y comprensible [...]

3. Después que los alumnos hayan analizado, discutido y observado el modelo, pueden comenzar a practicar la destreza. El profesor debe destacar y aprobar las ejecuciones correctas y tratar de evitar las inconvenientes. [...]

6. [...] darse privilegio a la forma correcta de ejecución y no destacar las faltas que puedan cometerse. [...] 
7. [...] la ejecución de las destrezas debe hacerse en forma automática y mecánica. $[\ldots]$

9. La distribución del tiempo y de los períodos de práctica ha de ser tal que asegure el máximo de mejoramiento con el mínimo de tiempo. [...] Esto significa una buena organización de la clase para conseguir los resultados que se desean en el tiempo dado 10. [...] la ejecución de las actividades con la máxima eficacia y facilidad [...]

11. Hay que ofrecerles en todo momento oportunidades para que critiquen sus propias performances $[\ldots]$

12. [...] ejercitar las destrezas fundamentales para asegurar la retención de las reacciones deseables. [...] Los alumnos deben conocer los objetivos que se tratan de conseguir y permitírseles que dirijan su propia práctica dentro de lo posible [...]" (PREOBRAYENSKY, 1954, p. 11-14).

El involucramiento del alumno en la autocrítica de su propia ejecución, y para ello el conocimiento del modelo, las conductas y objetivos deseables a alcanzar es parte central de esta perspectiva. La planificación detallada de las actividades de aprendizaje en función de los objetivos es fundamental. La centralidad en la práctica en tanto desarrollo de las actividades de aprendizaje era clave.

\section{Principios generales de toda buena práctica}

1. La práctica y la ejecución deben ir acompañadas por cierta satisfacción [...] Una manera de conseguirlo es organizar las destrezas fundamentales en forma de juegos simples de carreras de postas [...]

2. Los alumnos deben tener interés en las actividades que se están practicando. Para conseguirlo hay que hacerles comprender el significado de la ejercitación y que las actividades estén dentro del campo de sus experiencias [...]

4. Las destrezas deben practicarse en la misma forma que (...) se emplearán en [...] situación real $[\ldots]$

6. [...] atención a la ejecución del ejercitamiento [...] atienda todo el tiempo conciente y seriamente si desea mejorarlas $[\ldots]$

7. La práctica debe conducirse con viveza y precisión para reducir el tiempo que transcurre entre el estímulo y la respuesta. En una ejecución eficiente y hábil de las actividades motoras, la velocidad va unida a la exactitud. (PREOBRAYENSKY, 1954, p. 14-16).

Los juegos eran utilizados como estímulos positivos para obtener respuestas agradables y satisfacción en la actividad. El partir de experiencias pasadas ayudaba a captar el interés y la obtención de buenos resultados de aprendizaje. Los resultados eran evaluados en función de los objetivos planteados, así se cerraba el ciclo de planificación lineal, racional y técnica: establecimiento de objetivos, planificación y ejecución de actividades y evaluación de los resultados en función de los objetivos planteados. El "control y manejo" de este proceso circular era central en esta didáctica curricularizada.

En varias de las tesis, inspirada en la filosofía pragmática de raigambre anglosajona, se insistía en que la educación física era una de las mejores escuelas de formación para la vida, para el trabajo y que debía buscar en todo momento una utilidad práctica. Así se expresaba que la educación física debía tender "a la formación de sujetos honestos, capaces, utilitarios y con 
capacidad de trabajo muy amplia” (DE MARÍA, 1954, p. 22). Para esto se debía lograr "resultados utilitarios" y el profesor "deberá tender a lograr realizaciones utilitarias propias que bregarán por el logro de adelantos y progresos en la faz educativa que dirige" (DE MARÍA, 1954, p. 59).

En la didáctica curricularizada fue central la figura del profesor de educación física como un buen administrador, gestor y planificador de las actividades, la teoría de la administración tayloriana que esta discursividad trasladó desde el ámbito económico al pedagógico fue clave en su configuración. Así se expresan las diferentes características que debía tener una directora técnica a cargo de un equipo de básquetbol femenino, donde se destacaba la capacidad de organización y administración.

\begin{abstract}
Resumiendo, para tener éxito como directora técnica de un equipo se necesitan conocimientos pedagógicos, conocimientos técnicos, habilidad para escoger las jugadoras que son campeonas potenciales, voluntad para entrenar asidua y firmemente, una filosofía deportiva amplia, una personalidad adecuada, y capacidad para organizar y administrar. De todas estas cualidades tal vez la última sea más importante, porque ella da a la directora técnica lo necesario para coordinar todas las demás haciendo su trabajo coherente e inteligente. (PREOBRAYENSKY, 1954, p. 134).
\end{abstract}

\title{
CONSIDERACIONES FINALES
}

En este trabajo se abordó la formación del profesor de educación física desde la mirada peculiar de los estudiantes a punto de egresar, el análisis de sus tesis permitió conocer la selección y tamiz de conceptos, ideas, teorías y perspectivas relativas a la enseñanza, la educación y la educación física a lo largo de su formación. En cierta medida las tesis se constituyeron en un elemento síntesis y catalizador de la trayectoria formativa recorrida.

Gran cantidad de tesis al abordar las características de un buen profesor de educación física y los principios pedagógico-didácticos de la educación física, lo extraían en forma resumida o textual de los manuales del profesor Alberto Langlade. Esto da cuenta de la gran ascendencia que tuvo e imprimió a la formación, formateó modos de actuar, sentir y pensar de los estudiantes ${ }^{21}$.

Las tesis son expresión de las diversas circulaciones y traducciones de ideas en el campo de la educación física y sus ciencias auxiliares (fisiología, pedagogía, psicología entre las más importantes) tanto a nivel regional, europeo como norteamericano. Se citan con mayor

\footnotetext{
${ }^{21}$ El estudio de Torrón (2016) realiza diez entrevistas a profesores de educación física que dictaron clases en el ISEF y que fueron estudiantes en las décadas del 50 y 60 , y sus testimonios también realzan y dan cuenta de la importancia de la figura del profesor Alberto Langlade en su formación.
} 
frecuencia autores de Argentina, Brasil, Chile, Portugal, Francia, Suecia, Alemania, Estados Unidos. Esto da cuenta de la gran circulación de ideas y de una peculiar traducción local realizada fundamentalmente a través de los profesores a cargo de la formación, quienes viajaron con gran frecuencia en este período.

Con respecto a las principales nociones de educación y enseñanza presentes en los trabajos académicos de los estudiantes, el escolanovismo como pedagogía psicologizada y el tecnicismo como didáctica curricularizada tuvieron un peso predominante formando parte de una discursividad de la teoría de la enseñanza-aprendizaje.

El movimiento Escuela Nueva o Activa, con la complejidad de corrientes psicológicas y filosóficas que albergó en su seno, se configuró más cercano a la educación que a la didáctica, si bien adquirió componentes de la segunda. Fue caracterizado a través de las tesis en torno a los significantes naturaleza, integralidad, libertad, espontaneidad, centralidad en el alumno, partiendo de sus intereses y de su realidad, y de la idea de niño activo en oposición a pasivo realizando una dura crítica a la escuela tradicional y su trasmisión mecánica de conocimientos.

Varias tesis entendieron los principios generales de enseñanza de la educación física como máximas metodológicas prescriptivas para una buena enseñanza, como un conjunto de procedimientos determinados por las características orgánicas tanto mentales como corporales de los educandos, así se minimizaba o quedaba relegado el lugar del saber en la enseñanza. En ellos se hacía hincapié en cuatro aspectos:

1. en la gradación de la actividad física en grado de complejidad creciente al mejor estilo de una didáctica comeniana;

2. el desarrollo de principios pedagógicos escolanovistas, como el trabajo por centros de interés, la centración en el alumno, el partir de los deseos e intereses del grupo y el individuo;

3. el método pensado desde el desarrollo de las teorías psicológicas del aprendizaje y del desarrollo evolutivo, todos ellos articulados en una didáctica psicologizada;

4. la centración en el desarrollo fisiológico del cuerpo.

La didáctica curricularizada o tecnicista mostró sus mayores relieves en aquellas tesis que citaron en forma predominante literatura anglosajona, fundamentalmente de Norteamérica. La psicología conductista, fundamentalmente de Skinner, fue la base teórica más citada de esta perspectiva, mixturada con una filosofía de corte pragmático. Los principios de eficiencia y eficacia en la actividad física, la centración en la tarea o actividad de aprendizaje motor, la figura del profesor de educación física como un administrador o gestor del aprendizaje, la planificación por objetivos de conducta observable, la secuenciación y fragmentación de las actividades configurando progresiones de ejercicios, fueron de las principales contribuciones

Rev. Iberoam. Patrim. Histórico-Educativo, Campinas (SP), v. 4, n. 1, p. 122-143, jan.jun. 2018 
de esta discursividad inspirada en los principios de la planificación económica en serie tayleriana. Lo económico vuelve a guiar y conducir el trabajo pedagógico del profesor de educación física, por momentos mezclados y en otros soslayando aspectos espiritualistas o escolanovistas.

\section{REFERENCIAS}

ACHENBACH, Dina. La psicología del niño y la educación física. 1954. s/p. Tesis para la obtención de título de Profesor de Educación Física. ISEF. Montevideo: CNEF.

AISENSTEIN, Ángela; ELÍAS, L. Influencias de la YMCA en la cultura institucional del Instituto Nacional de Educación Física (INEF) Gral. Belgrano. Argentina, 1939 - 1967. XIII Congreso Iberoamericano de Historia de la Educación Latinoamericana, Montevideo, Uruguay, 28 febrero al 3 marzo 2018.

; FEGUIN, M. Diseño de sujetos morales, sanos y patriotas. Formación de profesores de Educación Física. Argentina, 1938-1967. Pedagogía y Saberes, Universidad Pedagógica Nacional, Facultad de Educación, n. 44, 2016, p. 9-20.

BEHARES, Luis. Didáctica Moderna: ¿más o menos preguntas, más o menos respuestas? In: . y Colombo (comps.) Enseñanza del saber y saber de la enseñanza. Montevideo: FHCE, 2005, p. 9-15.

BENTANCOR, Blanca. Educación y Educación Física. 1954. 93 f. Tesis para la obtención de título de Profesor de Educación Física. ISEF. Montevideo: CNEF.

BORDOLI, Eloisa. La Didáctica y lo didáctico. Del sujeto epistemológico al sujeto del deseo. In: BEHARES, Luis; COLOMBO, Susana (comps.). Enseñanza del saber y saber de la enseñanza. Montevideo: FHCE, 2005, p. 17-25.

BUSCH, Marta. Enseñanza del estilo crowl. 1954. 98 f. Tesis para la obtención de título de Profesor de Educación Física. ISEF. Montevideo: CNEF.

CARUSO, Marcelo. ¿Una nave sin puerto definitivo? Antecedentes, tendencias e interpretaciones alrededor del movimiento de la Escuela Nueva. In: ; Dussel, Inés; Pineau, Pablo (eds.). La escuela como máquina de educar. Tres escritos sobre un proyecto de la modernidad. Buenos Aires: Paidós, 2001, p. $93-131$.

CNEF Edufísica. Órgano de la Comisión Nacional de Educación Física. Segunda Época. Año 1, Número 1. Enero de 1955. Montevideo: CNEF, 64p.

CRAVIOTTO, Agustina; MALÁN, Paula. Entre la gimnasia y el deporte: la configuración de la educación física en el Uruguay (1911-1930). Políticas Educativas, Porto Alegre, v. 7, n.1, 2014, p. 32-42. Disponible en: <http://seer.ufrgs.br/index.php/Poled/article/view/50930/31699>. Acceso: 20 abr. 2018. 
DA SILVA, Giovana. A Associação Cristã de Moços e experiências de escolarização da Educação Física no Brasil: sujeitos, ideias e práticas acemistas em circulação. 2017. 236 f.Tesis Doctorado en Educación Faculdade de Educação, UFMG, Belo Horizonte.

; LINHALES, Meily. A Young Men's Christian Association - YMCA e a formação de diretores físicos: a preparação especializada em Chicago. XIII Congreso Iberoamericano de Historia de la Educación Latinoamericana Sociedad Uruguaya de Historia de la Educación, Montevideo, Uruguay, 28 febrero al 3 marzo 2018.

DE MARÍA, Hugo Raúl. Condiciones del profesor de Educación Física. 1954. 118 f. Tesis para la obten ción de título de Profesor de Educación Física. ISEF. Montevideo: CNEF.

DIAZ BARRIGA, Ángel. Didáctica. Aportes para una polémica. Buenos Aires: Aique, 1991, 63p.

DOGLIOTTI, Paola. Educación del cuerpo y discursividades en torno a la formación en educación física en Uruguay (1874-1948). Montevideo: Comisión Sectorial de Investigación Científica (CSIC) de laUniversidad de la República (Udelar), 2015. 259p.

; MALÁN, Paula. La influencia protestante y del movimiento de Springfield College en la educación del cuerpo y la educación física en el Uruguay. In: MARTÍNEZ MOCTEZUMA, Lucía. (coord.). Formando el cuerpo del ciudadano. Aportes para una historia de la educación física en Latinoamerica. México: Universidad Autónoma del Estado de Morelos: Morelos, Instituto del Deporte, 2015, p. 279 - 304.

INSTITUTO SUPERIOR DE EDUCACIÓN FÍSICA (ISEF). Reglamento del Instituto Superior de Educación Física. ISEF - CNEF (Comisión Nacional de Educación Física), Montevideo, 1952, 10p.

. Libro de registro de firmas de titulados en educación física. ISEF- Universidad de la República (Udelar), Montevideo, 2014.

LANGLADE, Alberto. Manual de Didáctica de la Gimnástica. Publicación N ${ }^{\circ}{ }^{1}$. ISEF CNEF, Montevideo, 1956.

LINHALES, Meily. Tránsito de sujetos y métodos norteamericanos: propuestas para la enseñanza de la educación física dentro y fuera de las escuelas brasileñas. In: MARTÍNEZ MOCTEZUMA, Lucía (coord.). Formando el cuerpo del ciudadano. Aportes para una historia de la educación física en Latinoamerica. México: Universidad Autónoma del Estado de Morelos: Morelos, Instituto del Deporte, 2015, p. 259 - 278.

MALÁN, Paula. Cristiandad Muscular y crisis del ethos valdense: Un estudio discursivo del ingreso del deporte a las Uniones Cristianas de Jóvenes de las colonias valdenses del Uruguay (1920 - 1970). 2017. 217 f.Tesis de Maestría en Educación Corporal. Facultad de Humanidades y Ciencias de la Educación. Universidad Nacional de la Plata.

MAUAD, Salvador. La Educación Física en un presente con miras a un futuro. 1953. 87 f. Tesis para la obtención de título de Profesor de Educación Física. ISEF. Montevideo: CNEF. 
MARKARIAN, Vania. Al ritmo del reloj: adolescentes uruguayos de los años cincuenta. In: BARRÁN, J. P.; CAETANO, G.; PORZECANSKI, T. (Dir.). Historias de la vida privada en el Uruguay. Tomo 3. Individuo y soledades 1920 - 1990. Uruguay: Taurus, 2004, p. 239 - 264.

MARTÍNEZ MOCTEZUMA, Lucía. El cambio hacia un modelo norteamericano de educación física: las Misiones Culturales en el México rural, 1923 - 1940. In: (Coord.). Formando el cuerpo del ciudadano. Aportes para una historia de la educación física en Latinoamerica. México: Universidad Autónoma del Estado de Morelos, Morelos, Instituto del Deporte, 2015, p. $305-328$.

PÊCHEUX, M. O Discurso. Estrutura ou acontecimento. Campinas: Pontes, 1990.

PREOBRAYENSKY, Irene. Técnica del basketball femenino. 1954. 148 f. Tesis para la obtención de título de Profesor de Educación Física. ISEF. Montevideo: CNEF.

SCARLATO, Inés. Plazas vecinales de cultura física (1913): una versión uruguaya de los playgroun ds norteamericanos. XIII Congreso Iberoamericano de Historia de la Educación Latinoamericana, Montevideo, Uruguay, 28 febrero al 3 marzo 2018.

TORRÓN, Ana. Gimnasia y Deporte en el Instituto Superior de Educación Física (19391973): su configuración y su enseñanza. 2015. 194 f. Tesis de Maestría en Enseñanza Universitaria, Comisión Sectorial de Enseñanza, Área Social, Universidad de la República, Montevideo.

TYLER, Ralph. Principios básicos del currículo. Buenos Aires: Troquel, 1973[1949].

VAZ FERREIRA, Carlos. La educación física. Anales de Educación Física de la CNEF, Montevideo, CNEF, Tomo III, 1, 1922 [1959] p. 33-51. 